\title{
Skipping Breakfast and Eating Breakfast Away From Home Were Prospectively Associated With Emotional and Behavioral Problems in 115,217 Chinese Adolescents
}

\author{
Wei-Jie Gong ${ }^{1,2}$, Daniel Yee-Tak Fong ${ }^{1}$, Man-Ping Wang ${ }^{1}$, Tai-Hing Lam², \\ Thomas Wai-Hung Chung ${ }^{3}$, and Sai-Yin $\mathrm{Ho}^{2}$ \\ ${ }^{1}$ School of Nursing, Li Ka Shing Faculty of Medicine, The University of Hong Kong, Hong Kong, China \\ ${ }^{2}$ School of Public Health, Li Ka Shing Faculty of Medicine, The University of Hong Kong, Hong Kong, China \\ ${ }^{3}$ Family \& Student Health Branch, Department of Health, Hong Kong, China
}

Received April 1, 2021; accepted May 31, 2021; released online June 19, 2021

\begin{abstract}
Background: Breakfast is deemed the most important meal of the day. We examined the prospective associations of breakfast habits with emotional/behavioral problems in adolescents and potential effect modification.

Methods: 115,217 Primary 6 students (United States Grade 6; mean age, 11.9; standard deviation [SD], 0.59 years) who attended the Student Health Service of Department of Health in Hong Kong in 2004/05, 2006/07, 2008/09 were followed till Secondary 6 (United States Grade 12). Emotional/behavioral problems were biennially examined using Youth Self-Report since Secondary 2 (United States Grade 8). Lifestyles were biennially examined using standardized questionnaires since Primary 6. Prospective associations of breakfast habit with emotional/behavioral problems and potential effect modification were examined using generalized estimating equations.

Results: Compared with eating breakfast at home, eating breakfast away from home was significantly associated with total emotional/behavioral problems and seven syndromes, including withdrawal, somatic complaints, anxiety/depression, thought problems, attention problems, delinquent behaviors, and aggressive behaviors (adjusted odds ratios [AORs] 1.22-2.04), while skipping breakfast showed stronger associations with the above problems and social problems (AORs 1.34-2.29). Stronger associations were observed in younger students for total and attention problems $(P<0.03)$ and in those with lower weight status for delinquent behaviors $(P=0.005)$.

Conclusions: Eating breakfast away from home and especially skipping breakfast were prospectively associated with adolescent emotional/behavioral problems. The associations weakened with increasing age for total emotional/behavioral and attention problems, and weakened with higher weight status for delinquent behaviors, highlighting the vulnerability of younger and underweight children. If the associations are causal, increasing home breakfast may reduce adolescent emotional/behavioral problems and benefit psychosocial health.
\end{abstract}

Key words: breakfast habit; emotional problems; behavioral problems; adolescent

Copyright $\odot 2021$ Wei-Jie Gong et al. This is an open access article distributed under the terms of Creative Commons Attribution License, which permits unrestricted use, distribution, and reproduction in any medium, provided the original author and source are credited.

\section{INTRODUCTION}

Adolescents are vulnerable to psychological problems with longterm implications on health and well-being. ${ }^{1}$ Half of all mental disorders are symptomatic by the age of 14 , although most of them remain undiagnosed and untreated until later in life. ${ }^{2}$ Worldwide, around $10-20 \%$ of children and adolescents are affected by mental health problems. ${ }^{3}$ Moreover, adolescents with aggressive and delinquent behaviors have a high risk of becoming adult offenders. ${ }^{4}$ Such emotional and behavioral problems in adolescents could be influenced by their lifestyles and health-related behaviors, ${ }^{5}$ including physical activity and dietary habits. ${ }^{6,7}$

Breakfast has been widely regarded as the most important meal of the day, especially for children and adolescents, ${ }^{8,9}$ providing energy and nutrients for their growing body after fasting overnight. ${ }^{10}$ Regular breakfast consumption promotes youth's nutritional status, weight maintenance, ${ }^{11,12}$ and cognitive performance. ${ }^{8,13}$ In contrast, poor breakfast habits, including skipping breakfast, may lead to the deficiency of many micronutrients that may not be compensated for by other meals. ${ }^{14-16}$ Poor breakfast habits can also cause metabolic inflexibility with higher postprandial insulin levels and increased fat oxidation, resulting in low-grade inflammation and impaired glucose homeostasis in the long run. ${ }^{16}$ Despite the importance of breakfast, $10-30 \%$ of adolescents from 33 countries and regions skipped breakfast, and the trend was increasing. ${ }^{17}$

Previous studies mostly focused on the benefits of breakfast on children's mental health. ${ }^{18,19}$ Only several cross-sectional studies 
have linked breakfast skipping to emotional and behavioral problems, including depression, school bullying, and stress symptoms. ${ }^{20-22}$ No longitudinal evidence has been reported.

Also, with easy access to fast food, more children eat breakfast away from home. ${ }^{23,24}$ Foods served away from home are typically high-sugar products with lower nutrition quality, ${ }^{25}$ which have been linked to emotional symptoms in children. ${ }^{26}$ However, whether eating breakfast away from home is prospectively associated with emotional and behavioral health remains unknown. Whether there are interactions among these associations is also unclear. We hypothesized that breakfast location and breakfast skipping were associated with adolescent psychosocial health. Therefore, we aimed to assess the prospective associations of breakfast skipping and eating breakfast away from home with emotional and behavioral problems using longitudinal data of Chinese adolescents in Hong Kong and to explore potential effect modifiers.

\section{METHODS}

\section{Design and subjects}

This was a territory-wide population-based longitudinal study. All subjects were participants of the Student Health Service (SHS) of the Department of Health, Hong Kong Special Administrative Region, China. The SHS provided free voluntary annual health assessments for all primary and secondary school students in 12 SHS centers, which covered all districts in Hong Kong. Students were invited to complete a standardized questionnaire on lifestyles in Primary 4 (P4 or United States Grade 4), P6, Secondary 2 (S2 or United States Grade 8), S4, and S6. They also completed the Youth Self-Report (YSR) for assessing emotional and behavioral problems in S2, S4, and S6. Most (97\%) students completed the Chinese version of YSR while the English version was also available. We obtained anonymous data from the SHS, with longitudinal data for each student linked using a unique identification number.

P6 students in the academic years of 2004/05, 2006/07, and 2008/09 were enrolled in this study and were tracked for 6 years until S6 in 2010/11, 2012/13, and 2014/15, respectively. P6 students in 2005/06 and 2007/08 were not included because they could not attend the SHS appointments in their S4 and S2, respectively, in 2009/10 when the SHS was engaged in the Human Swine Influenza Vaccination Programme. A total of 175,113 P6 students were included, corresponding to $74.8 \%$ of all P6 students in Hong Kong in the academic years of 2004/05, 2006/07, and 2008/09.27

The participants' parents/guardians gave written consent each year to enroll in the annual SHS health assessments, including use of the participants' data for research. ${ }^{28}$ The study protocol was approved by the Institutional Review Board of The University of Hong Kong/Hospital Authority Hong Kong West Cluster (Reference number: UW 19-206) and the Department of Health Ethics Committees (Reference number: L/M 66/2019).

\section{Measurements}

The outcomes were emotional/behavioral problems in S2, S4, and S6 using YSR, which was shown to be reliable and valid in Hong Kong children and adolescents. ${ }^{29,30}$ YSR consists of 112 items with a 3-point response scale (scores): not true (0), somewhat or sometimes true (1), and very true or often true (2). ${ }^{31}$ It has a total scale and eight syndrome subscales, with scores above the local threshold indicating the respective problems. A total score of over 71 in boys and 78 in girls indicates total emotional and behavioral problems. Threshold scores for the subscales (boys, girls) are: withdrawal $(9,10)$, somatic complaints $(9,10)$, anxiety/depression $(19,21)$, social problems $(10,10)$, thought problems $(8,8)$, attention problems $(13,13)$, delinquent behaviors $(9,8)$ and aggressive behaviors $(21,20) .{ }^{29}$ The thresholds were developed using local Hong Kong norms. ${ }^{32}$

Lifestyles were assessed in P6, S2, and S4 using a set of closed-ended items. The study factor of breakfast habit was assessed by the item 'I usually have breakfast at...' with four response options: (i) 'no breakfast at all' denoted skipping breakfast, (ii) 'home' denoted eating at home, (iii) 'fast-food stall/cafeteria/restaurant' and (iv) 'some other places' denoted eating breakfast away from home. The frequency and duration of extracurricular aerobic physical activities, such as ball sports, swimming, and running, were dichotomized as $<1$ or $\geq 1$ time/ week and $<60$ or $\geq 60$ minutes/week, respectively.

Sex, age, weight, height, and socioeconomic status were also obtained from the SHS. Weight (to the nearest $0.1 \mathrm{~kg}$ ) and height (to the nearest $0.1 \mathrm{~cm}$ ) were annually measured by well-trained healthcare staff following standard procedures. Socioeconomic status included parental educational level (primary or below, secondary, and tertiary) and parental occupation (unemployed, manual job, clerical/service industry, and managerial/professional job).

\section{Statistical analysis}

Body mass index (BMI) was calculated as weight $(\mathrm{kg})$ divided by squared height $\left(\mathrm{m}^{2}\right)$. According to the International Obesity Task Force standards, weight status was classified as underweight, normal, overweight and obese using age-sex-specific BMI cut-off values that correspond to BMI values of 18.5, 25.0, and 30.0 $\mathrm{kg} / \mathrm{m}^{2}$ at 18 years of age. ${ }^{33}$ To examine whether the included sample was representative, the characteristics of the participants were compared with those of the corresponding children and adolescent population in Hong Kong ${ }^{34}$ using Cohen's d for age, with values of $0.20,0.50$, and 0.80 denoting small, medium, and large effect sizes, respectively, ${ }^{35}$ and Cramer's $\mathrm{V}$ for categorical variables, with values of $0.10,0.30$, and 0.50 denoting small, medium, and large effect sizes, respectively. ${ }^{36}$

The characteristics of adolescents were compared among those eating breakfast at home, away from home, and skipping breakfast in P6 using the Kruskal-Wallis test and Chi-square test for continuous and categorical characteristics, respectively. The prospective associations of breakfast habit (eating breakfast at home, away from home, and skipping breakfast) with the outcomes were examined using generalized estimating equations (GEE) models with a logit link. Specifically, the main exposure was breakfast habit, which was time-dependent and measured in P6, S2, and S4, and the outcomes were the presence of total emotional/behavioral problems and the eight syndromes, which were measured in S2, S4, and S6. Unstructured correlation matrix was used to account for the extra-covariance among the longitudinal measurements. An adjustment was made for sex, parental educational level and parental occupation, year enrolled in P6, and time-dependent factors, including age, weight status, and frequency/duration of extracurricular physical activity in P6, S2, and S4. The Benjamini-Hochberg correction was used to account for multiple testing of different emotional and behavioral problems, ${ }^{37}$ while the Dunnett-Hsu method was used to adjust the 
$P$-values of multiple pairwise comparisons across breakfast habits. Adjusted odds ratios (AORs) with 95\% confidence intervals (CIs) were reported. Tests for trend across breakfast habits were performed by including breakfast habits as a threecategory ordinal variable (eating breakfast at home, away from home, and skipping breakfast as 1,2, and 3, respectively) in the respective GEE models. ${ }^{38}$ The moderating effects of sex, age, weight status, parental educational level, and parental occupation with breakfast habits were estimated on all the outcomes by incorporating the corresponding interaction terms. Statistically significant interactions were estimated using the ratio of odds ratios (RORs). A ROR greater than 1 indicates that the differences in the odds ratios increased between the compared breakfast habits. For statistically significant interactions, the predicted probabilities of outcomes across breakfast habits were estimated in the respective GEE models and plotted in separate lines across the moderators. To examine the slope of separate lines of breakfast habits, tests for trend were used by including the moderators as continuous variables in the respective GEE models. ${ }^{38}$

In addition, we estimated the mean and standard error of the total and subscale YSR scores across sex, grade, and breakfast habits, and their adjusted regression coefficients ( $\beta \mathrm{s}$ ) with $95 \%$ CIs using GEE models, adjusted for academic year enrolled in P6, parental educational level and occupation, and time-dependent factors in Primary 6 and Secondary 2 and 4, including weight status and frequency/duration of extracurricular physical activity, and mutually adjusted for each other. All analyses were conducted using the Statistical Analysis System (SAS Institute, Cary, NC, USA) 9.4 with a two-tailed significance level of 0.05 .

\section{RESULTS}

Among the 175,113 P6 students who attended the SHS service, 55,117 (31.5\%) with no records in S2, S4, or S6; $1,972(1.1 \%)$ with no breakfast habit data in P6; and 2,807 (1.6\%) with missing data of socioeconomics and/or frequency/duration of extracurricular physical activity in P6 were excluded. After removing them from the present analysis, 115,217 (65.8\%) students (girls $52.2 \%$; mean age 11.9; standard deviation [SD], 0.59 years) remained, with an average of 1.7 (SD, 0.73) follow-up records (range, 1 to 3 ). The differences of the characteristics between the included sample and the corresponding Hong Kong population were small, with all Cramer's $V \leq 0.10$ (eTable 1). The differences of the P6 characteristics between the included and excluded samples were also small, with Cohen's $d=0.16$ for age and all Cramer's V for categorical characteristics $\leq 0.10$ (eTable 2).

In $\mathrm{P} 6,84.5 \%, 8.7 \%$, and $6.8 \%$ of participants ate breakfast at home, away from home, and skipped it, respectively. Table 1 shows significant differences in sex, weight status, parental educational level, parental occupation, frequency and duration of physical activity, and year enrolled in P6. Compared with children who ate breakfast at home, those who ate breakfast away from home or skipped breakfast had a higher percentage of being overweight $(19.7 \%$ and $22.2 \%$ vs $15.8 \%)$ and obese $(4.6 \%$ and $5.4 \%$ vs $2.8 \%$ ), and a lower percentage of having tertiaryeducated parents $(11.4 \%$ and $10.6 \%$ vs $17.2 \%)$, parents working in managerial/professional position (18.6\% and $18.6 \%$ vs $24.3 \%$ ), and doing physical activity $\geq 1$ time/week $(74.2 \%$ and $66.1 \%$ vs $76.7 \%$ ) (all $P<0.001)$.
Table 2 shows that, compared with students who ate breakfast at home, those who ate away from home had significantly higher AORs of total emotional/behavioral problems (AOR 1.45) and seven syndromes, including withdrawal (AOR 1.22), somatic complaints (AOR 1.40), anxiety/depression (AOR 1.42), thought problems (AOR 1.27), attention problems (AOR 1.52), delinquent behaviors (AOR 2.04) and aggressive behaviors (AOR $1.55)(P=0.016$ to $<0.001)$ but not social problems (AOR 1.14; 95\% CI, 0.93-1.39, $P=0.21$ ). Skipping breakfast was associated with higher AORs of total emotional/behavioral problems (AOR $1.87)$ and all eight syndromes (AORs 1.34 to 2.29$)(P=0.008$ to $<0.01$ ). Compared with students who ate breakfast away from home, those who skipped breakfast had higher AORs of total emotional/behavioral problems (AOR 1.28) and three syndromes including somatic complaints (AOR 1.51), thought problems (AOR 1.31), and aggressive behaviors (AOR 1.34) $(P=0.04$ to $<0.001)$.

Only three interactions were statistically significant (Figure 1), including those between age and breakfast habit for total emotional/behavioral problems $(P=0.01)$ and attention problems $(P=0.03)$, as well as that between weight status and breakfast habit for delinquent behaviors $(P=0.005)$. Figure 1A shows that as students grew from 10 to 18 years of age, the predicted probabilities of total emotional/behavioral problems were stable for those who ate breakfast at home ( $P$ for trend $=$ 0.18 ) but decreased for those who ate breakfast away from home ( $P$ for trend $=0.002$ ) or skipped breakfast $(P$ for trend $=0.009)$. In general, the predicted probabilities of total emotional/ behavioral problems for eating breakfast at home were lower than those for eating breakfast away from home or skipping breakfast. However, the differences decreased (eating breakfast away from home: ROR $0.92 ; 95 \% \mathrm{CI}, 0.86-0.99, P=0.04$ and skipping breakfast: ROR $0.92 ; 95 \%$ CI, $0.85-1.00, P=0.02$ ) with increasing age. Figure 1B shows similar trends in the predicted probabilities of attention problems. Similarly, the predicted probabilities of attention problems for eating breakfast at home was lower than those for eating breakfast away from home or skipping breakfast. The difference with skipping breakfast decreased significantly (ROR 0.87 ; 95\% CI, 0.74-0.99, $P=$ 0.03 ) but that with eating breakfast away from home was not significant (ROR 0.91; 95\% CI, 0.82-1.02, $P=0.11$ ). Figure 1C shows that with higher weight status, the predicted probabilities of delinquent behaviors increased for eating breakfast at home ( $P$ for trend $<0.001$ ) but was stable for eating breakfast away from home $(P$ for trend $=0.31)$ and skipping breakfast $(P$ for trend $=0.47$ ). The predicted probabilities of delinquent behaviors for eating breakfast at home was generally lower than those for eating breakfast away from home or skipping breakfast. However, the difference decreased (eating breakfast away from home: ROR 0.69 ; 95\% CI, 0.53-0.90, $P<0.01$ and skipping breakfast: ROR 0.72 ; 95\% CI, $0.55-0.93, P=0.01)$ with higher weight status.

When taking the total and subscale YSR scores as continuous outcomes, as shown in eTable 3, girls had higher scores than boys for total emotional/behavioral problems and all subscale problems except for lower scores of delinquent behaviors (all $P \leq 0.04)$. Participants in higher grades had higher scores in total scores and the scores of withdrawal, somatic complaints, anxiety/ depression, attention problems, and delinquent problems (all $P<$ 0.001). Compared with eating breakfast at home, eating breakfast away from home and skipping breakfast predicted higher scores of total problems (adjusted $\beta$ s: 2.60 and 5.99, respectively) and all 
Table 1. Baseline characteristics of students by breakfast habits in Primary $6, n(\%)$

\begin{tabular}{|c|c|c|c|c|c|}
\hline \multirow[b]{2}{*}{ Characteristics } & \multirow[b]{2}{*}{$\begin{array}{c}\quad \text { All } \\
(n=115,217)\end{array}$} & \multicolumn{3}{|c|}{ Breakfast habits } & \multirow[b]{2}{*}{$P^{\mathrm{a}}$} \\
\hline & & $\begin{array}{l}\text { Eating at home } \\
\quad(n=97,405)\end{array}$ & $\begin{array}{c}\text { Eating away from home } \\
\qquad(n=9,994)\end{array}$ & $\begin{array}{l}\text { Skipping } \\
(n=7,818)\end{array}$ & \\
\hline Age, mean (SD), years & $11.9(0.59)$ & $11.9(0.59)$ & $12.0(0.59)$ & $12.0(0.65)$ & $<0.001$ \\
\hline Sex & & & & & $<0.001$ \\
\hline Male & $55,027(47.8)$ & $46,171(47.4)$ & $5,171(51.7)$ & $3,685(47.1)$ & \\
\hline Female & $60,190(52.2)$ & $51,234(52.6)$ & $4,823(48.3)$ & $4,133(52.9)$ & \\
\hline Weight status & & & & & $<0.001$ \\
\hline Underweight & $16,208(14.1)$ & $14,238(14.6)$ & $1,184(11.9)$ & $786(10.1)$ & \\
\hline Normal & $76,351(66.3)$ & $65,099(66.8)$ & $6,380(63.8)$ & $4,872(62.3)$ & \\
\hline Overweight & $19,041(16.5)$ & $15,337(15.8)$ & $1,969(19.7)$ & $1,735(22.2)$ & \\
\hline Obese & $3,617(3.1)$ & $2,731(2.8)$ & $461(4.6)$ & $425(5.4)$ & \\
\hline Parental educational level & & & & & $<0.001$ \\
\hline Primary or below & $13,953(12.1)$ & $11,595(11.9)$ & $1,286(12.9)$ & $1,072(13.7)$ & \\
\hline Secondary & $82,546(71.6)$ & $69,060(70.9)$ & $7,569(75.7)$ & $5,917(75.7)$ & \\
\hline Tertiary & $18,718(16.3)$ & $16,750(17.2)$ & $1,139(11.4)$ & $829(10.6)$ & \\
\hline Parental occupation & & & & & $<0.001$ \\
\hline Unemployed & $6,110(5.3)$ & $5,069(5.2)$ & $527(5.3)$ & $514(6.6)$ & \\
\hline Manual job & $39,392(34.2)$ & $33,340(34.2)$ & $3,447(34.5)$ & $2,605(33.3)$ & \\
\hline Clerical/service industry & $42,764(37.1)$ & $35,354(36.3)$ & $4,162(41.6)$ & $3,248(41.6)$ & \\
\hline Managerial/professional & $26,951(23.4)$ & $23,642(24.3)$ & $1,858(18.6)$ & $1,451(18.6)$ & \\
\hline Frequency of extracurricular physical activity & & & & & $<0.001$ \\
\hline$<1$ time/week & $27,962(24.3)$ & 25,736 (23.3) & $2,573(25.8)$ & $2,653(33.9)$ & \\
\hline$\geq 1$ time/week & $87,255(75.7)$ & $74,669(76.7)$ & $7,421(74.2)$ & $5,165(66.1)$ & \\
\hline Duration of extracurricular physical activity & & & & & $<0.001$ \\
\hline$<60$ minutes/week & $71,842(62.4)$ & $60,515(62.1)$ & $6,188(61.9)$ & $5,139(65.7)$ & \\
\hline$\geq 60$ minutes/week & $43,375(37.7)$ & $36,890(37.9)$ & $3,806(38.1)$ & $2,679(34.3)$ & \\
\hline \multicolumn{6}{|l|}{ Year in Primary 6} \\
\hline $2004 / 05$ & $39,230(34.1)$ & $33,435(34.3)$ & $3,267(32.7)$ & $2,528(32.3)$ & $<0.001$ \\
\hline 2006/07 & $40,934(35.5)$ & $34,543(35.5)$ & $3,494(35.0)$ & $2,897(37.1)$ & \\
\hline 2008/09 & $35,053(30.4)$ & $29,427(30.2)$ & $3,233(32.4)$ & $2,393(30.6)$ & \\
\hline
\end{tabular}

$\mathrm{SD}$, standard deviation.

${ }^{\text {a}}$ Based on Kruskal-Wallis test for age, and Chi-square test for other variables.

Table 2. The prospective associations of breakfast habits in Primary 6 , Secondary 2 and Secondary 4 with emotional and behavioral problems in Secondary 2, 4, and $6^{a}$

\begin{tabular}{|c|c|c|c|c|c|}
\hline Emotional/behavioral problems & $\begin{array}{l}P \text { for overall } \\
\text { association }^{b}\end{array}$ & $\begin{array}{l}\text { Eating away from home } \\
\text { vs Eating at home }\end{array}$ & $\begin{array}{l}\text { Skipping vs Eating } \\
\text { at home }\end{array}$ & $P$ for trend & $\begin{array}{l}\text { Skipping vs Eating away } \\
\text { from home }\end{array}$ \\
\hline The total emotional/behavioral problems & $<0.001$ & $1.45(1.30,1.62)^{\mathrm{e}}$ & $1.87(1.66,2.10)^{\mathrm{e}}$ & $<0.001$ & $1.28(1.11,1.48)^{\mathrm{d}}$ \\
\hline Withdrawal & $<0.001$ & $1.22(1.04,1.44)^{\mathrm{c}}$ & $1.52(1.27,1.82)^{\mathrm{e}}$ & $<0.001$ & $1.24(1.00,1.55)$ \\
\hline Somatic complaints & $<0.001$ & $1.40(1.19,1.65)^{\mathrm{e}}$ & $2.12(1.81,2.48)^{\mathrm{e}}$ & $<0.001$ & $1.51(1.23,1.85)^{\mathrm{e}}$ \\
\hline Anxiety/depression & $<0.001$ & $1.42(1.18,1.70)^{\mathrm{e}}$ & $1.70(1.40,2.06)^{\mathrm{e}}$ & $<0.001$ & $1.19(0.94,1.53)$ \\
\hline Social problems & $<0.001$ & $1.14(0.93,1.39)$ & $1.34(1.08,1.66)^{\mathrm{d}}$ & 0.009 & $1.18(0.89,1.54)$ \\
\hline Thought problems & $<0.001$ & $1.27(1.05,1.54)^{\mathrm{c}}$ & $1.67(1.37,2.04)^{\mathrm{e}}$ & $<0.001$ & $1.31(1.02,1.70)^{c}$ \\
\hline Attention problems & $<0.001$ & $1.52(1.28,1.80)^{\mathrm{e}}$ & $1.82(1.51,2.19)^{\mathrm{e}}$ & $<0.001$ & $1.19(0.95,1.50)$ \\
\hline Delinquent behaviors & $<0.001$ & $2.04(1.71,2.44)^{\mathrm{e}}$ & $2.29(1.89,2.79)^{\mathrm{e}}$ & $<0.001$ & $1.12(0.89,1.42)$ \\
\hline Aggressive behaviors & $<0.001$ & $1.55(1.29,1.85)^{\mathrm{e}}$ & $2.08(1.73,2.49)^{\mathrm{e}}$ & $<0.001$ & $1.34(1.06,1.70)^{c}$ \\
\hline
\end{tabular}

${ }^{a}$ Adjusted odds ratios (95\% confidence interval) with eating breakfast at home or eating breakfast away from home as the reference group, adjusted for sex, parental educational level and occupation status, enrolled year in Primary 6 and age, weight status and frequency/duration of extracurricular physical activity in Primary 6, Secondary 2 and 4.

${ }^{\mathrm{b}}$ The overall $P$-values tested about the null hypothesis of no difference in the odds of an emotional/behavioral problem across all breakfast habits. $P$-values were adjusted by the Benjamini-Hochberg correction.

${ }^{\mathrm{c}} P<0.05$.

${ }^{\mathrm{d}} P<0.01$.

${ }^{\mathrm{e}} P<0.001$.

syndrome scores (adjusted $\beta \mathrm{s}: \quad 0.07-0.66$ and $0.31-1.21$, respectively) (all $P<0.001$ ).

\section{DISCUSSION}

This is the first longitudinal study showing that both eating breakfast away from home and skipping breakfast were associated with emotional and behavioral problems in adolescents. Eating breakfast away from home was associated with higher odds of total emotional/behavioral problems and seven syndromes except for social problems, and skipping breakfast was associated with higher odds of total emotional/behavioral 

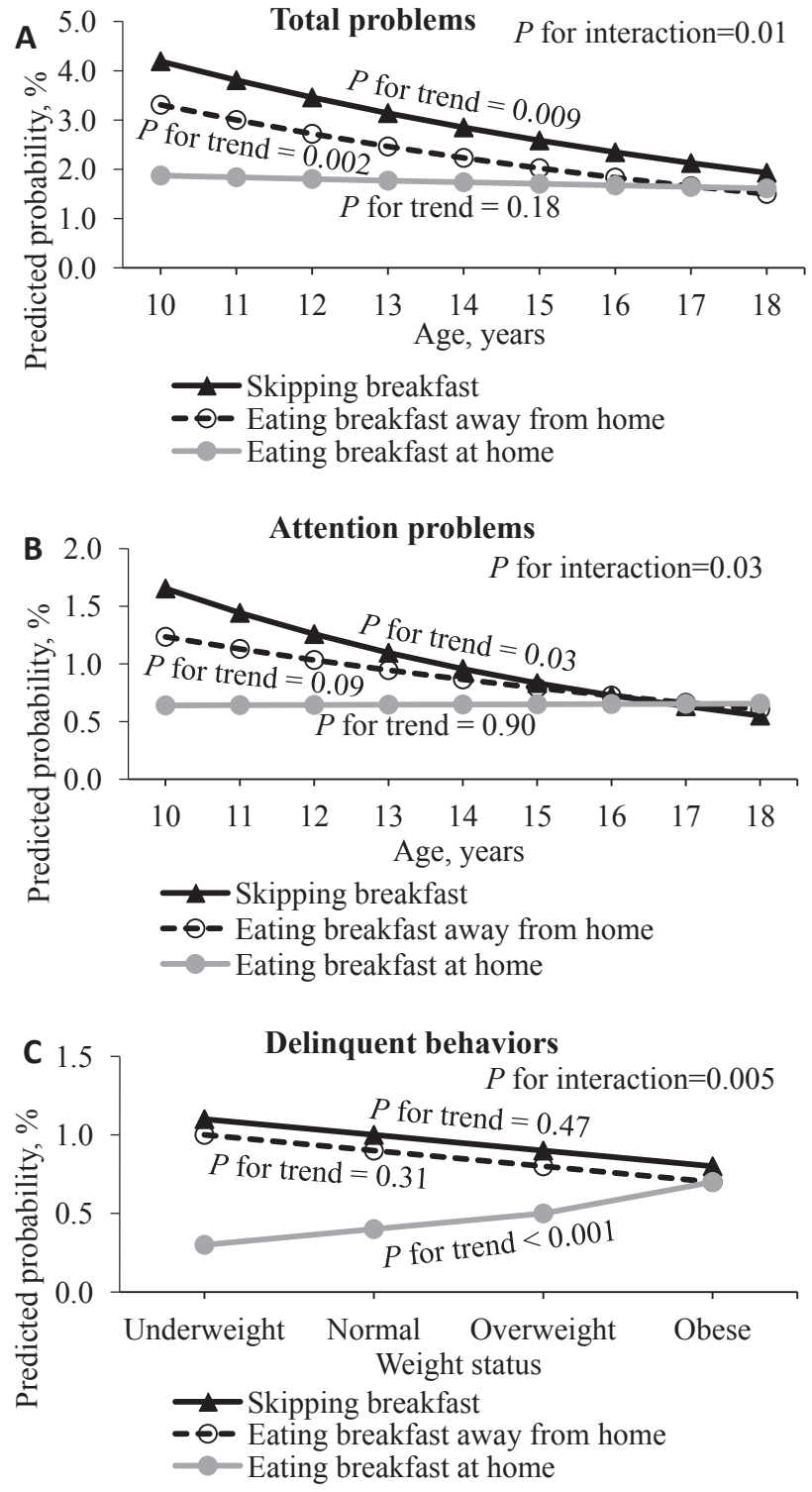

Figure 1. The predicted probability (\%) of $(\mathbf{A})$ the total emotional/behavioral problems and (B) attention problems by breakfast habits and age, as well as (C) delinquent behaviors by breakfast habits and weight status. ${ }^{a}$

${ }^{a}$ All predicted probabilities were estimated using generalized estimating equations with a logit link, with having the corresponding problems as 1 , adjusted for sex, parental educational level and parental occupation, year enrolled in P6 and timedependent factors, including age, weight status and frequency/duration of extracurricular physical activity.

problems and all eight syndromes. Age modified the associations of breakfast habits with the total emotional/behavioral problems and attention problems, with stronger associations at younger ages. Weight status modified the association between breakfast habits and delinquent behaviors, with the predicted probabilities of delinquent behaviors slightly increased with higher weight status only for eating breakfast at home but was stable for eating breakfast away from home or skipping breakfast.
Among the three breakfast habits, eating breakfast at home was the most beneficial one for adolescent psychosocial health. Compared with eating breakfast at home, eating breakfast away from home predicted higher odds of total emotional/behavioral problems and several syndromes. Considering that home meals were reported to have superior nutritional quality to those served away from home in Western countries, ${ }^{25,39}$ breakfast nutrition could be one possible reason, but this may differ across cultures, which warrants detailed dietary assessment of local breakfast, including portion size, food variety, and nutrition quality. Meanwhile, even for the same food, eating at home may result in better emotional reinforcement than eating away from home. ${ }^{39}$ Home meals in post-meal affective states were found to have greater emotional reinforcing values than meals away from home. $^{3}$ This may start from the parent-child attachment relationship since the early stage of life. When trusting and caring parents provide food to their children, the secure home environment could help establish a secure attachment, ${ }^{39}$ which is important to the development of children's psychosocial health. Besides, adolescents who ate away from home or skipped breakfast might have parents who are busier or less caring, with less involvement in preparing breakfast meals and therefore have less parental monitoring and family communication, resulting in higher risks of emotional and behavioral problems.

Compared with eating breakfast away from home, skipping breakfast was prospectively associated with total emotional/ behavioral problems and several syndromes. There are several possible reasons. Mood regulation is a complex result of a series of different neurochemical pathways, each requiring several nutrients to supply the necessary metabolites, such as serotonin and dopamine. ${ }^{40,41}$ Nutrition supplementations in school children, including multivitamins, minerals, and $n-3$ fatty acids, have shown to be effective in treating depression and attention deficits $^{42-44}$ and reducing institutional violence and antisocial behaviors. ${ }^{45}$ Compared with children eating breakfast away from home, habitual breakfast skippers were more likely to have a deficiency in these essential micronutrients that produce the involved neurotransmitters. ${ }^{14-16}$ These micronutrient deficiencies could cause impaired brain function, attention deficits, and poorer cognitive performance, ${ }^{46}$ which subsequently lead to different syndromes of emotional and behavioral problems. Also, low frequency of eating breakfast was associated with low family functioning, including worse relations with parents, lower quality of family communication, and less family support. ${ }^{47}$ Furthermore, breakfast skippers tend to have other health-compromising lifestyles, including sedentary behaviors, smoking, and other unhealthy dietary habits, ${ }^{48,49}$ which may have combined effects on adolescent emotional and behavioral problems. More evidence is needed to unravel the underlying mechanisms.

We have also reported, for the first time, the interaction between breakfast habits with weight status on delinquent behaviors (Figure 1C). With higher weight status, eating breakfast away from home and skipping breakfast showed stable predicted probabilities of delinquent behaviors ( $P$ for trend $\geq 0.31$ ), whereas eating breakfast at home showed increasing predicted probabilities ( $P$ for trend $<0.001)$. Insufficient nutrition intake from breakfast may be one of possible reasons for the adverse effects from unhealthy breakfast habits. ${ }^{25,39}$ However, as overweight and obese children tend to be over-nourished, they would be less susceptible to inadequate nutritional intake due 
to eating breakfast away from home or skipping breakfast. Therefore, their risks of delinquent behaviors showed smaller changes when they did not eat breakfast at home. Breakfast prepared at home may have higher nutrition quality, which would have more benefits to children at a lower weight status. In other words, eating breakfast at home had minimal, if any, beneficial effects in obese children for reducing their risks of developing delinquent behaviors. Further prospective or qualitative evidence considering other related factors, such as weight satisfaction and peer relationship, would be valuable.

Notably, we also found weaker associations of breakfast habits with total emotional/behavioral problems and attention problems with increasing age, indicating the vulnerability of younger children in psychosocial health when having unfavorable breakfast habits. Younger children are still in the early stage of physical and psychosocial development, so they may be more sensitive to the influence of breakfast habit. Special attention should be given to younger children to develop healthy dietary habits, although skipping breakfast was less prevalent in them. ${ }^{50}$ There have been tremendous increases in the consumption of food away from home and skipping breakfast globally. ${ }^{23,24,50}$ To promote these children to eat breakfast at home, the health awareness of their parents and families should be improved.

According to the guide that an OR of 2.0 is recommended as a 'practically' significant effect, ${ }^{51}$ the associations of eating breakfast away from home with delinquent behaviors, and the associations of skipping breakfast with somatic complaints, delinquent behaviors, and aggressive behaviors remain 'practically' significant, while the associations with withdrawal and social problems were relatively small. Our findings suggest that the associations of unhealthy breakfast habits may manifest more in adolescent externalizing behaviors (corresponding to delinquent and aggressive behaviors in YSR), ${ }^{29}$ such as lying/ cheating, stealing, running away, arguing, fighting, and attacking, rather than inner distress. These findings support previous studies linking unhealthy diets with externalizing behaviors in children. ${ }^{52,53}$ We have first shown the associations of breakfast habits with somatic complaints in adolescents. Somatic complaints, withdrawal, and anxiety/depression comprised internalizing problems in YSR. ${ }^{29}$ Somatic complaints, including feeling dizzy or tired, having headaches, and vomiting, were found to vary by culture, with somatic expressions of distress more common in Asian people and affective expressions of distress more common in Western people. ${ }^{54,55}$ Further studies are warranted to explore the underlying mechanisms. Moreover, this study benefited from the large sample size to have statistically significant findings from associations with small effect size, which, however, should not be neglected due to the clustering of unhealthy behaviors. For example, insufficient sleep, pathological internet use, and online risky behaviors were found to be common and associated with breakfast skipping in Asian children, ${ }^{56,57}$ and they were all found to link with psychosocial problems. ${ }^{58,59}$ More evidence is needed on whether such cluster may have cumulative adverse effects on mental health in young people.

This study had some limitations. First, self-reported questionnaires may be subject to recall bias and social expectations bias. However, they are most viable in large-scale studies with a long follow-up. Second, as nutrition quality is often lower in breakfast served away from home than that in home-made breakfast, the behavioral pattern of purchasing breakfast away from home but eating it at home should be considered in future studies, which can examine the influences of home environment. Third, although school breakfast programs are widely provided in Western countries, school breakfast could not be included in this study due to the lack of such programs in Hong Kong. Fourth, despite our efforts to minimize residual confounding, there might remain other confounding factors relating to both breakfast habits and emotional/behavioral problems. For example, breakfast skipping may be associated with other unhealthy lifestyles, such as insufficient sleep and sedentary behaviors, ${ }^{56,57}$ and unfavorable family environment, such as poor family relationship and nonintact family. Therefore, only promoting eating breakfast at home could not solve all adolescent emotional/behavioral problems. Further studies are warranted to find effective psychosocial health promotion measures in children and adolescents. Lastly, there were noticeable missing values in this large-scale territory-wide study, and the attendance rate in follow-ups decreased with higher academic years. However, the small differences between the characteristics of our sample with the census data of the corresponding Hong Kong population indicated good representativeness (eTable 1), and only small effect sizes were found for the differences in baseline characteristics between the students who were and were not included in this study (eTable 2).

\section{Conclusions}

Eating breakfast away from home and especially skipping breakfast were prospectively associated with more adolescent emotional/behavioral problems. The associations weakened with increasing age for total and attention problems, and weakened with higher weight status for delinquent behaviors, highlighting the vulnerability of younger and underweight children. If the associations are causal, increasing home breakfast may reduce adolescent emotional/behavioral problems and benefit psychosocial health.

\section{ACKNOWLEDGEMENTS}

We thank all colleagues and staff at the Student Health Service of the Department of Health for their assistance and collaboration. The study was funded by the Health and Health Services Research Fund, Food and Health Bureau, Hong Kong SAR, China [grant number 05060781].

Funding source: The study was funded by the Health and Health Services Research Fund, Food and Health Bureau, Hong Kong SAR [grant number 05060781]. The funder was not involved in the conduct of the research and/or preparation of the article, in study design; in the collection, analysis and interpretation of data; in the writing of the report; and in the decision to submit the article for publication.

Contribution' statement: Dr Gong conceived and conducted the analysis and drafted the initial manuscript. Dr Fong developed the study methodology, assisted in interpreting the data and drafting the manuscript. Dr Wang and Prof Lam critically reviewed the manuscript for important intellectual content. Dr Chung contributed to data collection and critically reviewed the manuscript. Dr Ho designed the original study, acquired the data and critically reviewed the manuscript. All authors approved the final manuscript as submitted and agree to be accountable for all aspects of the work.

Conflicts of interest: None declared. 


\section{APPENDIX A. SUPPLEMENTARY DATA}

Supplementary data related to this article can be found at https:// doi.org/10.2188/jea.JE20210081.

\section{REFERENCES}

1. Call KT, Riedel AA, Hein K, McLoyd V, Petersen A, Kipke M. Adolescent health and well-being in the twenty-first century: a global perspective. J Res Adolesc. 2002;12(1):69-98.

2. Kessler RC, Berglund P, Demler O, Jin R, Merikangas KR, Walters EE. Lifetime prevalence and age-of-onset distributions of DSM-IV disorders in the National Comorbidity Survey Replication. Arch Gen Psychiatry. 2005;62(6):593-602.

3. Kieling $\mathrm{C}$, Baker-Henningham $\mathrm{H}$, Belfer $\mathrm{M}$, et al. Child and adolescent mental health worldwide: evidence for action. Lancet. 2011;378(9801):1515-1525.

4. Wallinius M, Delfin C, Billstedt E, Nilsson T, Anckarsäter H, Hofvander B. Offenders in emerging adulthood: school maladjustment, childhood adversities, and prediction of aggressive antisocial behaviors. Law Hum Behav. 2016;40(5):551-563.

5. Brassai L, Piko BF, Steger MF. Meaning in life: is it a protective factor for adolescents' psychological health? Int J Behav Med. 2011; 18(1):44-51.

6. Biddle SJ, Asare M. Physical activity and mental health in children and adolescents: a review of reviews. Br J Sports Med. 2011;45(11): 886-895.

7. Jacka FN, Kremer PJ, Leslie ER, et al. Associations between diet quality and depressed mood in adolescents: results from the Australian Healthy Neighbourhoods Study. Aust N Z J Psychiatry. 2010;44(5):435-442.

8. Adolphus K, Lawton CL, Champ CL, Dye L. The effects of breakfast and breakfast composition on cognition in children and adolescents: a systematic review. Adv Nutr. 2016;7(3):590S-612S.

9. Turner L, Chaloupka FJ. Continued promise of school breakfast programs for improving academic outcomes: breakfast is still the most important meal of the day. JAMA Pediatr. 2015;169(1):13-14.

10. Deshmukh-Taskar PR, Nicklas TA, O’Neil CE, Keast DR, Radcliffe JD, Cho S. The relationship of breakfast skipping and type of breakfast consumption with nutrient intake and weight status in children and adolescents: the National Health and Nutrition Examination Survey 1999-2006. J Am Diet Assoc. 2010;110(6): 869-878.

11. Tin SP, Ho SY, Mak KH, Wan KL, Lam TH. Breakfast skipping and change in body mass index in young children. Int $\mathrm{J}$ Obes (Lond). 2011;35(7):899-906.

12. Fayet-Moore F, Kim J, Sritharan N, Petocz P. Impact of breakfast skipping and breakfast choice on the nutrient intake and body mass index of Australian children. Nutrients. 2016;8(8):487.

13. Hoyland A, Dye L, Lawton CL. A systematic review of the effect of breakfast on the cognitive performance of children and adolescents. Nutr Res Rev. 2009;22(2):220-243.

14. Affenito SG. Breakfast: a missed opportunity. J Am Diet Assoc. 2007;107(4):565-569.

15. Giménez-Legarre N, Miguel-Berges ML, Flores-Barrantes P, Santaliestra-Pasías AM, Moreno LA. Breakfast characteristics and its association with daily micronutrients intake in children and adolescents-a systematic review and meta-analysis. Nutrients. 2020; 12(10):3201.

16. Nas A, Mirza N, Hägele F, et al. Impact of breakfast skipping compared with dinner skipping on regulation of energy balance and metabolic risk. Am J Clin Nutr. 2017;105(6):1351-1361.

17. Monzani A, Ricotti R, Caputo M, et al. A systematic review of the association of skipping breakfast with weight and cardiometabolic risk factors in children and adolescents. What should we better investigate in the future? Nutrients. 2019;11(2):387.

18. O'Sullivan TA, Robinson M, Kendall GE, et al. A good-quality breakfast is associated with better mental health in adolescence. Public Health Nutr. 2009;12(2):249-258.
19. Murphy JM, Pagano ME, Nachmani J, Sperling $P$, Kane S, Kleinman RE. The relationship of school breakfast to psychosocial and academic functioning: cross-sectional and longitudinal observations in an inner-city school sample. Arch Pediatr Adolesc Med. 1998;152(9):899-907.

20. Sampasa-Kanyinga H, Roumeliotis $\mathrm{P}$, Farrow CV, Shi YF. Breakfast skipping is associated with cyberbullying and school bullying victimization. A school-based cross-sectional study. Appetite. 2014;79:76-82.

21. Tajik E, Latiffah AL, Awang H, et al. Unhealthy diet practice and symptoms of stress and depression among adolescents in Pasir Gudang, Malaysia. Obes Res Clin Pract. 2016;10(2):114-123.

22. Lien L. Is breakfast consumption related to mental distress and academic performance in adolescents? Public Health Nutr. 2007; 10(4):422-428.

23. Liu M, Kasteridis P, Yen ST. Breakfast, lunch, and dinner expenditures away from home in the United States. Food Policy. 2013;38:156-164.

24. Pingali P. Westernization of Asian diets and the transformation of food systems: implications for research and policy. Food Policy. 2007;32(3):281-298.

25. Taveras EM, Berkey CS, Rifas-Shiman SL, et al. Association of consumption of fried food away from home with body mass index and diet quality in older children and adolescents. Pediatrics. 2005; 116(4):e518-e524.

26. Kohlboeck G, Sausenthaler S, Standl M, et al; GINI-plus and LISAplus Study Groups. Food intake, diet quality and behavioral problems in children: results from the GINI-plus/LISA-plus studies. Ann Nutr Metab. 2012;60(4):247-256.

27. Hong Kong annual digest of statistics (Census and Statistics Department, Hong Kong SAR) (2005, 2007 and 2009).

28. Student Health Service. Enrolment forms and related information. Department of Health, The Government of Hong Kong SAR, China. Updated 31 August 2020. Accessed 21 April, 2021. https://www. studenthealth.gov.hk/english/resources/resources_forms/resources_ forms.html.

29. Leung PW, Kwong SL, Tang CP, et al. Test-retest reliability and criterion validity of the Chinese version of CBCL, TRF, and YSR. J Child Psychol Psychiatry. 2006;47(9):970-973.

30. Ivanova MY, Achenbach TM, Rescorla LA, et al. The generalizability of the youth self-report syndrome structure in 23 societies. J Consult Clin Psychol. 2007;75(5):729-738.

31. Achenbach TM. Manual for the teacher's report form and 1991 profile. Univ Vermont/Department Psychiatry; 1991.

32. Leung P, Lee C, Ho T, Hung S, Tang C. Achenbach Hong Kong norm: YSR profile for Hong Kong boys \& girls. Unpublished manuscript The Chinese University of Hong Kong. Hong Kong, China 1998.

33. Cole TJ, Bellizzi MC, Flegal KM, Dietz WH. Establishing a standard definition for child overweight and obesity worldwide: international survey. BMJ. 2000;320(7244):1240-1243.

34. Hong Kong 2006 population by-census (Hong Kong Census and Statistics Department, Hong Kong Special Administrative Region Government Hong Kong, China) (2006).

35. Lakens D. Calculating and reporting effect sizes to facilitate cumulative science: a practical primer for $t$-tests and ANOVAs. Front Psychol. 2013;4:863.

36. Cohen J. A power primer. Psychol Bull. 1992;112(1):155-159.

37. Benjamini Y, Hochberg Y. Controlling the false discovery rate: a practical and powerful approach to multiple testing. J R Stat Soc Series B Stat Methodol. 1995;57(1):289-300.

38. Szklo M, Nieto FJ. Epidemiology: beyond the basics. Jones \& Bartlett Publishers; 2014.

39. Lu J, Faber A, Dubé L. An evidence-based approach to the nutritional quality of home meals: Exploring emotional reinforcement and attachment style as underlying mechanisms. Diet Quality. Springer; 2013:27-40.

40. Hariri AR, Holmes A. Genetics of emotional regulation: the role of the serotonin transporter in neural function. Trends Cogn Sci. 2006; 10(4):182-191. 
41. Dunlop BW, Nemeroff CB. The role of dopamine in the pathophysiology of depression. Arch Gen Psychiatry. 2007;64(3): 327-337.

42. Eby GA, Eby KL. Rapid recovery from major depression using magnesium treatment. Med Hypotheses. 2006;67(2):362-370.

43. Leung BM, Wiens KP, Kaplan BJ. Does prenatal micronutrient supplementation improve children's mental development? A systematic review. BMC Pregnancy Childbirth. 2011;11(1):12.

44. Hurt EA, Arnold LE, Lofthouse N. Dietary and nutritional treatments for attention-deficit/hyperactivity disorder: current research support and recommendations for practitioners. Curr Psychiatry Rep. 2011;13(5):323-332.

45. Schoenthaler SJ, Bier ID. The effect of vitamin-mineral supplementation on juvenile delinquincy among American schoolchildren: a randomized, double-blind placebo-controlled trial. J Altern Complement Med. 2000;6(1):7-17.

46. Fanjiang G, Kleinman RE. Nutrition and performance in children. Curr Opin Clin Nutr Metab Care. 2007;10(3):342-347.

47. Pedersen TP, Holstein BE, Damsgaard MT, Rasmussen M. Breakfast frequency among adolescents: associations with measures of family functioning. Public Health Nutr. 2016;19(9):1552-1564.

48. Keski-Rahkonen A, Kaprio J, Rissanen A, Virkkunen M, Rose RJ. Breakfast skipping and health-compromising behaviors in adolescents and adults. Eur J Clin Nutr. 2003;57(7):842-853.

49. Tin SPP, Ho SY, Mak KH, Wan KL, Lam TH. Lifestyle and socioeconomic correlates of breakfast skipping in Hong Kong primary 4 schoolchildren. Prev Med. 2011;52(3-4):250-253.

50. Voráčová J, Sigmund E, Sigmundová D, Kalman M. Changes in eating behaviours among Czech children and adolescents from 2002 to 2014 (HBSC Study). Int J Environ Res Public Health. 2015; 12(12):15888-15899.
51. Ferguson CJ. An effect size primer: a guide for clinicians and researchers. Methodological issues and strategies in clinical research, 4th ed. American Psychological Association; 2016:301-310.

52. Oddy WH, Robinson M, Ambrosini GL, et al. The association between dietary patterns and mental health in early adolescence. Prev Med. 2009;49(1):39-44.

53. Liu J, Raine A, Venables PH, Mednick SA. Malnutrition at age 3 years and externalizing behavior problems at ages 8,11 , and 17 years. Am J Psychiatry. 2004;161(11):2005-2013.

54. Ryder AG, Yang J, Zhu X, et al. The cultural shaping of depression: somatic symptoms in China, psychological symptoms in North America? J Abnorm Psychol. 2008;117(2):300-313.

55. Kim JHJ, Tsai W, Kodish T, Trung LT, Lau AS, Weiss B. Cultural variation in temporal associations among somatic complaints, anxiety, and depressive symptoms in adolescence. J Psychosom Res. 2019;124:109763.

56. Gong QH, Li H, Zhang XH, Zhang T, Cui J, Xu GZ. Associations between sleep duration and physical activity and dietary behaviors in Chinese adolescents: results from the Youth Behavioral Risk Factor Surveys of 2015. Sleep Med. 2017;37:168-173.

57. Yamada M, Sekine M, Tatsuse T, Asaka Y. Prevalence and associated factors of pathological Internet use and online risky behaviors among Japanese elementary school children. J Epidemiol. 2020;31:537-544.

58. Wang W, Du X, Guo Y, et al. The associations between sleep situations and mental health among Chinese adolescents: a longitudinal study. Sleep Med. 2021;82:71-77.

59. Kojima R, Sato M, Akiyama Y, et al. Problematic Internet use and its associations with health-related symptoms and lifestyle habits among rural Japanese adolescents. Psychiatry Clin Neurosci. 2019; 73(1):20-26. 